3. N. Dunford and B. J. Pettis, Linear operators on summable functions, Trans. Amer. Math. Soc. vol. 47 (1940) pp. 323-392.

4. O. Nikodym, Sur une généralisation des intégrales de M. Radon, Fund. Math. vol. 15 (1930) pp. 137-179.

5. R. S. Phillips, Integration in convex linear topological space, Trans. Amer. Math. Soc. vol. 48 (1940) pp. 516-540.

6. C. E. Rickart, Integration in a convex linear topological space, Trans. Amer. Math. Soc. vol. 52 (1942) pp. 498-521.

7. F. Riesz, Untersuchen über Systeme integrierbar Funktionen, Math. Ann. vol. 69 (1910) pp. 449-497.

8. S. Saks, Theory of the integral, Warsaw, 1937.

UNIVERSITY OF WISCONSIN

\title{
A NOTE ON CORRELATION FUNCTIONS AND STABILITY IN DYNAMICAL SYSTEMS ${ }^{1}$
}

\section{R. PUTNAM}

1. In the system of differential equations

$$
x^{\prime}=f(x),
$$

in which $x=\left(x_{1}, \cdots, x_{n}\right)$ and $f(x)=\left(f_{1}, \cdots, f_{n}\right)$ is of class $C^{1}$, suppose that

$$
\operatorname{div} f=\partial f_{1} / \partial x_{1}+\cdots+\partial f_{n} / \partial x_{n}=0
$$

throughout a compact, invariant space, $\Omega$, of (finite) positive $v$-measure. It will be supposed that $\Omega$ is the closure of an open set and that $v$ refers to the ordinary $n$-dimensional Lebesgue volume measure in the $x$-space consisting of points $P=x$.

If $g(t)$ denotes any function of class $(L)$ on every finite $t$-interval of $-\infty<t<\infty$, then $M_{t}[g(t)]$ will be defined as

$$
M_{t}[g(t)]=\lim (2 T)^{-1} \int_{-T}^{T} g(t) d t, \quad T \rightarrow \infty,
$$

in case the limit exists. In view of the measure-preserving assumption (2), it follows from the Birkhoff ergodic theorem that if $f=f(P)$ denotes any function of class $\left(L^{2}\right)$ on $\Omega$, then the correlation function

$$
c_{P}(s)=M_{t}\left[f\left(P_{t+8}\right) \bar{f}\left(P_{t}\right)\right]
$$

(which depends on $f$ ) exists as a continuous function of $s$ on $-\infty<s$ $<\infty$ for almost all points $P$ of $\Omega$, where $P=x(0)$ and $P_{t}=x(t)$ denotes

Received by the editors February 22, 1954.

1 This work was supported in part by the National Science Foundation research grant (NSF-G481). 
a solution of (1) through the point $P$; cf. $[6$, p. 805]. Furthermore, according to a result of Wiener, there exists a uniquely determined monotone function $\sigma_{P}(\lambda)=\sigma_{P}^{f}(\lambda)$ satisfying $\sigma_{P}(-\infty)=0, \sigma_{P}(\lambda-0)$ $=\sigma_{P}(\lambda)$ such that

$$
c_{P}(s)=\int_{-\infty}^{\infty} e^{i s \lambda} d \sigma_{P}(\lambda)
$$

holds for all points $P$ for which (3) exists (hence, almost everywhere on $\Omega)$; cf. [6, p. 797]. Next, let $F(s)$ be defined by

$$
F(s)=\int_{\Omega} f\left(P_{s}\right) \bar{f}(P) d v, \quad-\infty<s<\infty .
$$

It follows from an integration over $\Omega$ of both sides of the equation (3) that $F(s)=\int_{\Omega} c_{P}(s) d v$, and hence, from (4), that

$$
F(s)=\int_{-\infty}^{\infty} e^{i s \lambda} d \Sigma(\lambda)
$$

where $\Sigma(\lambda)$ denotes the monotone function

$$
\Sigma(\lambda)=\int_{\Omega} \sigma_{P}(\lambda) d v
$$

cf. [6, pp. 813-814]. It should be noted that the functions (4) and (6) are almost periodic $\left(B^{2}\right)$; cf. [6, p. 798], and [1].

A solution $x=x(t)$ of (1) will be called stable (with respect to $\Omega$ ) if stability is meant in the Minding-Dirichlet or Liapounoff sense; thus, for every $\epsilon>0$ there exists a $\delta=\delta_{\epsilon}>0$ such that $|x(t)-y(t)|<\epsilon$, $-\infty<t<\infty$, whenever $y(t)$ denotes a solution of (1) lying in $\Omega$ and satisfying $|x(0)-y(0)|<\delta$. See [2] and [4].

The following theorem will be proved:

$\left({ }^{*}\right)$ Let $f(P)$ denote a continuous function on the invariant space, $\Omega$, of the type considered above (so that $\Omega$ is compact and identical with the closure of an open set, and hence is of positive measure), and suppose that $P_{t}^{*}$ denotes a stable path of (1). Then, if $f\left(P_{t}^{*}\right)$ is almost periodic $\left(B^{2}\right)$, its frequencies are contained in the set of frequencies of the almost periodic $\left(B^{2}\right)$ function $F(s)$ defined by (5), that is, in the point spectrum of the function $\Sigma(\lambda)$, which depends on $f$ but is independent of the particular stable path $P_{t}^{*}$ under consideration, defined by (6) (or (7)).

As a consequence of $\left({ }^{*}\right)$ one obtains the following

COROLlary. If, in addition to the assumptions of $\left({ }^{*}\right)$, it is assumed that the function $F(S)$ of (5) satisfies 


$$
F(s) \rightarrow 0
$$$$
\text { as }|s| \rightarrow \infty \text {, }
$$

and if $f\left(P_{t}^{*}\right)$ is uniformly almost periodic (in the sense of Bohr), then $f\left(P_{t}^{*}\right) \equiv 0$ for $-\infty<t<\infty$.

In fact, according to $\left[6\right.$, p. 797] $M_{s}\left[e^{-i s \lambda} F(s)\right]$ exists and equals $\Sigma(\lambda+0)-\Sigma(\lambda-0)$. But, by (8), this difference is zero and the assertion of the last corollary now readily follows from $\left(^{*}\right)$.

It is interesting to note that, under the assumptions of $\left({ }^{*}\right)$ relating to $f(P)$ and $\Omega$, the limit

$$
\lim T^{-1} \int_{0}^{T} f\left(P_{t}^{*}\right) d t, \quad \text { as } T \rightarrow \infty(\text { or }-\infty),
$$

exists whenever $P_{t}^{*}$ is a stable (not necessarily almost periodic $\left(B^{2}\right)$ ) path [4]. Moreover, relation (8) (which represents a type of statistical independence [3, p. 67]) implies that $f$ is ergodic, so that $\lim T^{-1} \int_{0}^{T} f\left(P_{t}\right) d t$, as $T \rightarrow \infty$ (or $-\infty$ ), exists, and is zero (hence $\left.\int_{\Omega} f(p) d v=0\right)$, almost everywhere on $\Omega$; loc. cit. pp. 68-69. Thus, since $P_{t}^{*}$ is stable, each of the limits of (9) is zero (cf. the argument used in [4]) and so $M_{t}\left[f\left(P_{t}^{*}\right)\right]=0$. According to the theorem $\left({ }^{*}\right)$ however, if, in addition to (8), it is also assumed that $f\left(P_{t}^{*}\right)$ is uniformly almost periodic (in the sense of Bohr), then necessarily $f\left(P_{t}^{*}\right) \equiv 0,-\infty<t$ $<\infty$.

It is noteworthy that in $\left(^{*}\right)$ it is not assumed that the stable path, say $P_{t}^{*}$, is dense in the space $\Omega$. Thus, it is surprising that the frequencies of each of the $n$ components $x_{k}=x_{k}\left(P_{t}^{*}\right)$ of a stable almost periodic $\left(B^{2}\right)$ path are already determined by (more precisely, must be contained in) the set of frequencies of the corresponding almost periodic $\left(B^{2}\right)$ function $F_{k}(s)=\int_{\Omega} x_{k}\left(P_{s}\right) x_{k}(P) d v$, each being an integral taken over the entire "phase space" $\Omega$.

The theorem $\left({ }^{*}\right)$ is similar, in certain respects, to that given by Wiener and Wintner [6, p. 814]. In the present case, however, while the content of the assertion is restricted to continuous functions $f(P)$ on stable paths $P_{t}^{*}$, the hypothesis involves only the function $f(P)$ (and not the entire flow itself, $P_{t}$, of the system (1)).

For other results dealing with the connection between stability and almost periodicity, see [2] and [4].

2. Proof of $\left(^{*}\right)$. If $\lambda$ is a fixed (real) number, then $M_{t}\left[e^{-i \lambda t} f\left(P_{t}\right)\right]$ exists almost everywhere on $\Omega$; cf. [6, p. 804]. Since $P_{t}^{*}$ is stable, it readily follows that $M_{t}\left[e^{-i \lambda t} f\left(P_{t}^{*}\right)\right]$ exists for every $\lambda$. In fact, if this limit failed to exist for the stable path $P_{t}^{*}$, it would also fail to exist for all points $P$ sufficiently near $P^{*}$ (hence, in view of the assumptions 
on $\Omega$, on a subset of $\Omega$ of positive $v$-measure) in contradiction with the Birkhoff ergodic theorem; cf. [4].

Next, let $\lambda=\lambda_{0}$ belong to the frequency spectrum of $f\left(P_{t}^{*}\right)$, so that $M_{t}\left[e^{-i \lambda_{0} t} f\left(P_{t}^{*}\right)\right] \neq 0$. Then $M_{t}\left[e^{-i \lambda_{0} t} f\left(P_{t}\right)\right] \neq 0$ (again, cf. [4]) for almost all points $P$ in some neighborhood of $P^{*}$. In view of the inequality $\left|M_{t}\left[e^{-i \lambda_{0} t} f\left(P_{t}\right)\right]\right|^{2} \leqq \sigma_{P}\left(\lambda_{0}+0\right)-\sigma_{P}\left(\lambda_{0}-0\right)([6$, p. 799$] ;$ note that $f\left(P_{t}\right)$ need not be almost periodic $\left.\left(B^{2}\right)\right)$, it follows that $\sigma_{P}\left(\lambda_{0}+0\right)$ $-\sigma_{P}\left(\lambda_{0}-0\right)>0$ on some of set positive $v$-measure. According to (7), however, $\quad \Sigma(\lambda+0)-\Sigma(\lambda-0)=\int_{\Omega}\left[\sigma_{P}(\lambda+0)-\sigma_{P}(\lambda-0)\right] d v, \quad$ and so $\Sigma\left(\lambda_{0}+0\right)-\Sigma\left(\lambda_{0}-0\right)>0$; thus, $\lambda=\lambda_{0}$ is in the point spectrum of $\Sigma(\lambda)$. This completes the proof of $\left({ }^{*}\right)$.

\section{REFERENCES}

1. A. S. Besicovitch, Almost periodic functions, Cambridge, 1932.

2. P. Hartman and A. Wintner, Integrability in the large and dynamical stability, Amer. J. Math. vol. 65 (1943) pp. 273-278.

3. A. I. Khinchin, Mathematical foundations of statistical mechanics, New York, Dover.

4. C. R. Putnam, Stability and almost periodicity in dynamical systems, Proc. Amer. Math. Soc. vol. 5 (1954) pp. 352-356.

5. N. Wiener, The Fourier integral and certain of its applications, Cambridge, 1933.

6. N. Wiener and A. Wintner, On the ergodic dynamics of almost periodic systems, Amer. J. Math. vol. 63 (1941) pp. 794-824.

Purdue University 